Article

\title{
Concentrations of Carotenoids and Tocopherols in Breast Milk from Urban Chinese Mothers and Their Associations with Maternal Characteristics: A Cross-Sectional Study
}

\author{
Yong Xue ${ }^{1}$, Esther Campos-Giménez ${ }^{2, *}$, Karine Meisser Redeuil ${ }^{2}$, Antoine Lévèques ${ }^{2}$, \\ Lucas Actis-Goretta ${ }^{2}$, Gerard Vinyes-Pares ${ }^{3}$, Yumei Zhang ${ }^{1, *}$ (D), Peiyu Wang ${ }^{1}$ \\ and Sagar K. Thakkar ${ }^{2}$ \\ 1 Department of Nutrition \& Food Hygiene, School of Public Health, Peking University Health Science Center, \\ No. 38 Xueyuan Road, Haidian District, Beijing 100191, China; xueyong_pku@bjmu.edu.cn (Y.X.); \\ wpeiyu138@163.com (P.W.) \\ 2 Nestlé Research Center, Nestec, Vers-chez-les-blanc, CH-1000 Lausanne 26, Switzerland; \\ Karine.Meisser@rdls.nestle.com (K.M.R.); Antoine.Leveques@rdls.nestle.com (A.L.); \\ lucas.actisgoretta@rdls.nestle.com (L.A.-G.); sagar.thakkar@rdls.nestle.com (S.K.T.) \\ 3 Nestlé Research Center Beijing, Building E-F, No. 5 Dijin Road, Haidian District, Beijing 100095, China; \\ Gerard.VinyesPares@nestle.com \\ * Correspondence: campos-gimenez@rdls.nestle.com (E.C.-G.); zhangyumei@bjmu.edu.cn (Y.Z.); \\ Tel.: +86-10-8280-1575-63 (Y.Z.)
}

Received: 4 October 2017; Accepted: 7 November 2017; Published: 9 November 2017

\begin{abstract}
Milk composition remains the best estimate of infant requirements. The aims of this study were to quantify carotenoids and tocopherols in human milk from healthy Chinese mothers, and to explore their associations with lactation stage, region, socio-economic and obstetric characteristics, and dietary intake. Human milk was obtained from 509 healthy mothers, and concentrations of carotenoids and tocopherols were analyzed by Ultra High Performance Liquid Chromatography. The mothers' socio-economic and obstetric characteristics and dietary intake through a single 24-h dietary recall were evaluated. The median concentrations $(\mu \mathrm{g} / 100 \mathrm{~mL})$ of each component of 0-4 days, 5-11 days, 12-30 days, 31-60 days, 61-120 days, and 121-240 days postpartum were respectively as follows: $\beta$-carotene $8.0,2.8,2.1,1.7,1.9,1.8$; $\beta$-cryptoxanthin $6.2,3.4,2.4,1.7,1.8$, 2.1; lutein 5.7, 7.0, 2.2, 2.9, 2.8, 3.7; lycopene 6.3, 2.5, 1.8, 1.4, 1.4, 1.5; zeaxanthin 1.0, 1.4, 0.8, 0.8, 1.0, 1.1; $\alpha$-tocopherol 645, 382, 239, 206, 212, 211; $\gamma$-tocopherol 68, 63, 70, 73, 68, 88. The levels of those components varied significantly among different lactation stages and presented regional differences. Associations of carotenoid contents with maternal education, delivery mode, and present body mass index were found in multivariate analyses. These results suggested that lactation stage, region, and socio-economic and obstetric factors were associated with human milk concentrations of carotenoids and tocopherols in healthy Chinese mothers.
\end{abstract}

Keywords: breast milk; carotenoids; tocopherols; colostrum; lactation stage; cross-sectional study

\section{Introduction}

According to the World Health Organization, exclusive breastfeeding is recommended for the first six months of life [1], the period within which breast milk is the sole source of nutrition, providing all necessary nutrients to maintain health and permit normal growth. Thereafter, complementary feeding should be introduced while breastfeeding continues up to two years of age or beyond, so that breast milk is still a significant source of nutrients, at least in some parts of the world [2]. The recommended 
micronutrient intake for infants is currently based on the amounts provided by human milk from well-nourished women [3,4]; although this has been questioned by some authors, given the high variability observed among individuals [5,6]. Vitamin A is present in breast milk in the forms of preformed retinol (as retinyl esters), but also present as provitamin A carotenoids ( $\alpha$-carotene, $\beta$-carotene and $\beta$-cryptoxanthin). Since infants are born with very low reserves of vitamin $A$ in the liver regardless of the mother's nutritional status [3], they rely entirely on breast milk to support growth and build up liver storage. Vitamin $\mathrm{E}$ is a family of eight naturally occurring compounds sharing a common structure, and breast milk contains primarily $\alpha$-tocopherol, followed by smaller amounts of $\gamma$ - and $\beta$-tocopherol. Vitamin $\mathrm{A}$ and vitamin $\mathrm{E}$ are required in many essential metabolic functions for the growing infant $[7,8]$.

Given the importance of all these compounds for the newborn and the specific risk of fat-soluble vitamin deficiency in lactating women and breastfed children [9,10], the need to determine their concentrations in breast milk and how they depend on external or internal factors has been identified. A large number of reports in the composition of milk from Chinese mothers have focused on macronutrients [11-13], minerals [11,14], and fatty acids [14-20], while only a few studies report fat-soluble vitamin or carotenoid values [21-23]. Shi et al. [22] provide data on the vitamin content of milk from Chinese mothers from Inner Mongolia, which might be difficult to extrapolate to the whole country, partially due to the low number of data points and partially because it represents only a part of the country. The multinational study of Canfield et al. [21] showed differences in carotenoid patterns between countries, reflecting that in each one, the dietary carotenoid supply had no indication on the geographical origin of all samples, although this might be limited. Another multinational study [23] showed that regardless of the difficulty to detect trends due to high individual variability, some carotenoids presented clear differences between countries, which confirmed previous results, but also presented the same drawbacks related to the limited geographical variability of the samples. Thus, considering the geographical extension and multivariate lifestyles and diet in different parts of China, it would be necessary to study the carotenoid and tocopherol compositions of milk from Chinese women from different regions through lactation.

The changes in milk composition depending on different factors, such as stage of lactation or duration of the feeding [24-26], maternal diet, supplementation, and nutritional status [27-35] have been demonstrated. In recent years, association studies have found other potential factors influencing the concentrations of vitamins in breast milk, including maternal socio-economic [36-38], obstetric, or physiological factors [24,35-37,39,40]. Indeed, maternal socio-economic and obstetric factors are changing in China, such as the rate of cesarean delivery, which increased from $3.4 \%$ in 1988 to $39.3 \%$ in 2008 [41] and to 54.5\% in 2011 [42]; as well as the increase in inappropriate gestational weight gain (GWG). The latter is partly due to over nutrition and the rise of different dietary habits [43], which may have an impact on the micronutrient status of lactating women and the composition of their milk. Therefore, it is deemed necessary to research the associations of carotenoids and tocopherols in breast milk with maternal characteristics, and obstetric and nutritional factors in China.

The aims of this study were (1) to determine the composition of carotenoids and tocopherols in breast milk from healthy mothers from urban areas of China along lactation (zero to eight months) (2) to evaluate their interregional differences; and (3) to explore associations with nutrient intake. In addition, the associations with maternal socio-economic and obstetric characteristics, such as age, offspring gender, education, household income, delivery mode, body mass index (BMI), and GWG, were investigated. This study is part of the larger initiative Maternal Infant Nutrition Growth (MING) study. 


\section{Materials and Methods}

\subsection{Background of Participants}

The Maternal Infant Nutrition Growth (MING) study was a cross-sectional study designed to investigate the dietary and nutritional status of pregnant women, lactating mothers, and young children aged from birth up to three years living in urban areas of China. In addition, the human milk composition of Chinese lactating mothers was characterized. The study was conducted between 2011 and 2012. Three cities (Beijing is located in Northern China; Suzhou: Eastern China; Guangzhou: Southern China) were chosen for the characterization of human milk according to the geographical location and status of economic development. In each city, one grade A hospital and one maternal and child care hospital were randomly selected, and mothers at lactation period 0 to 240 days were randomly selected based on child registration information. Subject in the period 0-5 days were recruited at the grade A hospital, and subjects in the period 6-11 days and 12-30 days were contacted by phone to join the study, whereas other subjects were recruited at the maternal and child care hospital; if participation was dismissed, a replacement was made. Recruitment and milk sampling, as well as baseline data collection, were done on separate days.

A stratified sampling of 540 lactating mothers in 0-4 days, 5-11 days, 12-30 days, 31-60 days, 61-120 days, and 121-240 days postpartum was obtained in the MING study. Eligibility criteria included women between 18-45 years of age giving birth to a single, healthy, full-term gestation, and exclusive breastfeeding at least until four months. Exclusion criteria included gestational diabetes, hypertension, cardiac diseases, or acute communicable diseases. Lactating women who had nipple or lacteal gland diseases, had been using hormones in the last three months, experienced postpartum depression, or had insufficient language skills to understand the study questionnaires were also excluded. The study was approved by the Medical Ethics Research Board of Peking University (No. IRB00001052-11042). Written informed consent was obtained from all subjects participating in the study. The study was also registered in ClinicalTrials.gov with the number identifier NCT01971671.

In this cross-sectional study, carotenoids and tocopherols were quantified in 509 breast milk samples collected at different stages from early to late lactation in healthy Chinese women from three different cities (Beijing: $n=151$; Guangzhou: $n=180$; Suzhou: $n=178$ ). Figure 1 displays the recruitment flowchart from eligibility to sample analysis.

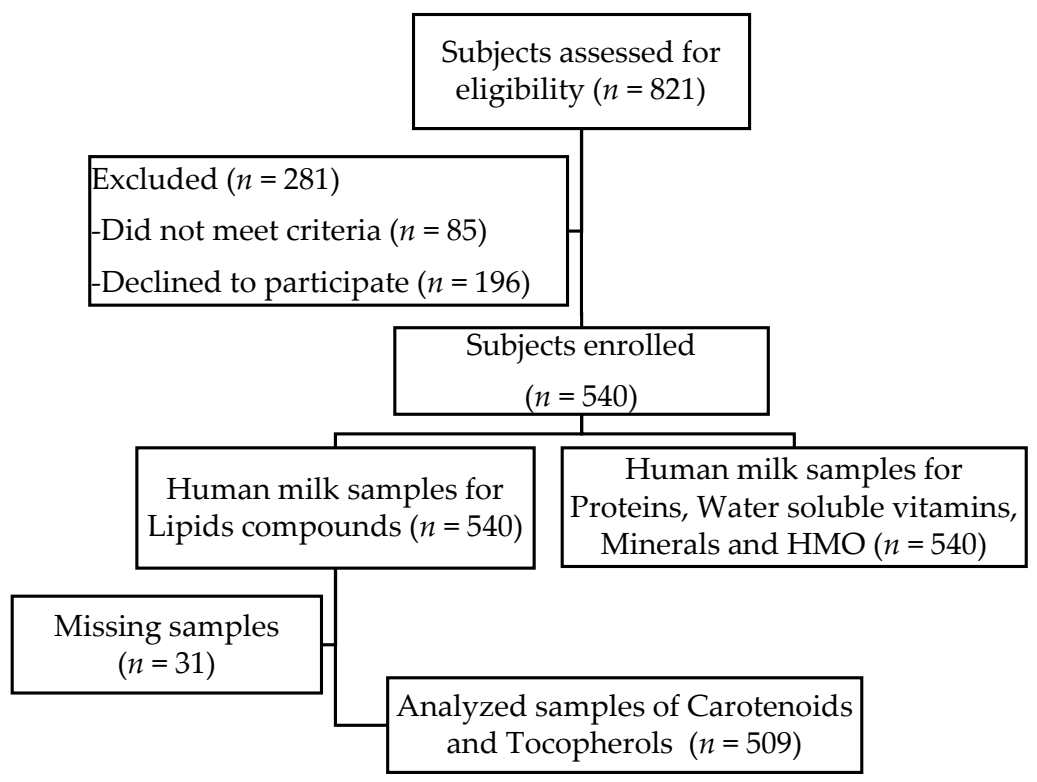

Figure 1. Study flow chart subjects enrolled. 


\subsection{Data Collection}

All subjects completed a structured questionnaire, which included socio-economic and lifestyle aspects of the mother such as household income, maternal education, and age. Self-reported weight at the beginning and at the end of pregnancy, the number of gestational weeks at delivery, and delivery mode were also recorded. Additionally, a physical examination evaluated basic anthropometric parameters including height and weight, which were explored to calculate the current body mass index (BMI), and BMI < 18.5, 18.5-24.9, 25.0-29.9, and $\geq 30 \mathrm{~kg} / \mathrm{m}^{2}$ were defined as underweight, normal weight, overweight, and obese, respectively. These data was obtained to calculate the gestational weight gain (GWG). According to the guidelines from the Institute of Medicines (IOM) in the United States [44], on average underweight women gain $12.5-18 \mathrm{~kg}$, normal weight women gain $11.5-16 \mathrm{~kg}$, overweight women gain 7-11.5 kg, and obese women gain 5-9 kg respectively; inadequate, adequate, and excessive weight gain were confirmed.

Through face-to-face interviews, data collection was performed during the day of the human milk sample collection. Additionally, subjects were contacted by phone and were asked to clarify the date of birth and gender of their baby, since the data was not included in the initial questionnaires.

\subsection{Dietary Assessment}

Dietary intake was assessed using a single 24-h dietary recall. Trained interviewers asked lactating mothers about all foods, beverages, and supplements consumed on the previous day, and then recorded this information in the questionnaire. To estimate the amount of foods and beverages consumed, a picture booklet of common foods consumed in China and measurement aids were used. Details about meals eaten out and food ingredients of homemade foods were also asked and recorded. Additionally, information on the use of dietary supplements was collected, including the name and brand of the supplement, and the amount. After revision of questionnaires, food records were entered in a database, and individual intakes of vitamin $\mathrm{A}$, total carotenoids, retinol, vitamin $\mathrm{E}$, and $\alpha$-tocopherol were processed with a food composition database created for this study that included data from Chinese Food Composition (CFC) tables 2004 \& 2009 [45,46], the Japanese Food Composition (JFC) tables 2005 [47], and branded products and supplements from China. In total, it contained the information of 1773 foods with 36 nutrients. Finally, we also compiled nutritional information from 75 dietary supplements sold in China.

\subsection{Sample Collection}

Breast milk sampling was standardized for all subjects, and was performed in a dim light room in hospitals without direct sunlight exposure. An electric pump (Horigen HNR/X-2108ZB, Xinhe Electrical Apparatuses Co., Ltd., Guangzhou, China) was used to collect the milk. Breasts of the subjects were emptied by the mother herself between 6-7 a.m. After the mothers had breakfast (7-8 a.m.), the samples were collected at the second feeding in the morning (9-11 a.m.) to avoid circadian influence on the outcomes. A single full breast was emptied by trained investigators into a new feeding bottle. We attempted to collect the milk from the same (right) breast, but milk was also collected from the left side when a steady stream of milk was not possible for some mothers. After gently up-down shaking for 10 times, an aliquot of $15 \mathrm{~mL}$ for colostrum and $40 \mathrm{~mL}$ for the subsequent time points was secured for characterization purposes. The rest of the milk was returned to the mother for feeding to the infant. Each sample was distributed in $1 \mathrm{~mL}$ clear polypropylene tubes under the dim light on ice, labeled with subject information, stored at $-80^{\circ} \mathrm{C}$, and then transported to the Nestlé Research Center, Lausanne (Switzerland) for analysis within six months of collection.

\subsection{Sample Preparation}

Briefly, $5 \mu \mathrm{L}$ of ethanol containing butylated hydroxytoluene (BHT) $(79 \mathrm{~g} / \mathrm{L})$ and $10 \mu \mathrm{L}$ of an aqueous solution of deferoxamine mesylate $(10 \mathrm{mg} / \mathrm{mL})$ to prevent oxidation, followed by $4 \mathrm{~mL}$ 
methanol, and $1 \mathrm{~mL}$ aqueous solution of potassium hydroxide $(\mathrm{KOH})(30 \% w / w)$ were added successively to $1 \mathrm{~mL}$ of milk into a $15-\mathrm{mL}$ tube. After mixing, the tube was placed for $30 \mathrm{~min}$ in a shaking water bath at $37^{\circ} \mathrm{C}$ for saponification. The samples were then cooled down on ice, and $5 \mathrm{~mL}$ of hexane containing $350 \mathrm{mg} / \mathrm{L} \mathrm{BHT}$ was added and mixed vigorously for $30 \mathrm{~s}$. Then, the tubes were centrifuged at $2500 \mathrm{rpm}$ for $10 \mathrm{~min}$ at $4{ }^{\circ} \mathrm{C}$, and the upper organic phase transferred to a clean $15-\mathrm{mL}$ tube by means of a glass Pasteur pipette. The liquid/liquid extraction process was repeated, and the organic phases combined in the same tube. Once completely dried under nitrogen at room temperature, the residue was dissolved in $70 \mu \mathrm{L}$ of dioxane/ethanol $(1 / 1, v / v)$ to ensure full recovery, and $70 \mu \mathrm{L}$ acetonitrile were finally added to better match initial mobile phase composition. The samples were centrifuged at $2500 \mathrm{rpm}$ for $10 \mathrm{~min}$ at room temperature to clarify extracts, and transferred into adapted low volume Ultra High Performance Liquid Chromatography (UHPLC) vials before analysis. The whole sample preparation and analysis were carried out in a blind laboratory under lights equipped with UV filers to prevent photosensitive analytes from degrading.

\subsection{Sample Analysis}

All the compounds ( $\alpha$-tocopherol, $\gamma$-tocopherol, $\beta$-carotene, $\beta$-cryptoxanthin, lutein, lycopene, and zeaxanthin were determined using a Waters Acquity UHPLC ${ }^{\circledR}$ system (Waters, Milford, MA, USA) equipped with a $2.1 \mathrm{~mm} \times 150 \mathrm{~mm}$ Waters Acquity UHPLC ${ }^{\circledR} \mathrm{HSS}$ T3 column, $100 \AA$ (particle diameter, $1.8 \mu \mathrm{m}$ ) placed in a column oven set at $35^{\circ} \mathrm{C}$, while autosampler was set at $20^{\circ} \mathrm{C}$.

A $5 \mu \mathrm{L}$-aliquot of the final extract was injected into the analytical system. The binary gradient eluting system pumped the mobile phase at a flow rate of $0.4 \mathrm{~mL} / \mathrm{min}$. Solvent A was a solution of ammonium acetate $0.05 \mathrm{M}$ in water, and solvent $B$ was a mixture of acetonitrile/diethyl ether/methanol $(76 / 9 / 15, w / w / w)$. The eluting gradient program was: 0-20 $\mathrm{min}, 75 \% \mathrm{~B} ; 20-22 \mathrm{~min}, 78 \% \mathrm{~B} ; 22-22.1 \mathrm{~min}$, $80 \%$ B; $22.1-30 \mathrm{~min}, 100 \%$ B; 30-42 $\mathrm{min}, 100 \%$ B; $42-42.1 \mathrm{~min}, 75 \%$ B; $42.1-55 \mathrm{~min}, 75 \%$ B. Quantification was performed by external calibration using pure standards. Concentration of standards was determined by spectrophotometry with corrections made for chromatographic purity. Carotenoids were detected and quantified using ultra violet (UV) at different wavelengths (lycopene, $472 \mathrm{~nm}$; $\beta$-carotene, $\beta$-cryptoxanthin, lutein, and zeaxanthin, $450 \mathrm{~nm}$; reference wavelength: $560 \pm 80 \mathrm{~nm}$ ), while $\alpha$-tocopherol and $\gamma$-tocopherol were detected and quantified by fluorescence $\left(\lambda_{\text {excitation }}: 298 \mathrm{~nm}\right.$, $\lambda_{\text {emission }}: 328 \mathrm{~nm}$ ). The standard calibration curve for each compound was constructed by plotting the response (peak area) versus the concentration using a weighted linear regression model.

A quality control (QC) sample consisting in pooled human milk with established analyte concentrations was analyzed in duplicate every 14 samples during analysis campaign. A typical series of analysis included 28 samples. Each analytical series was validated by evaluating accuracy on each QC if at least $67 \%$ of QC samples were within $20 \%$ of their respective nominal values.

Method performance was evaluated during method validation by analyzing pooled breast milk spiked with standard mixtures in duplicate $(k=2)$ on six different days $(n=6)$. Statistical analysis showed good intra-day and inter-day variability, being both below 15\% (expressed as the coefficient of variation). Average recovery rates were respectively $90.0 \%, 94.0 \%, 93.3 \%, 73.3 \%$, and $75.6 \%$ for $\beta$-carotene, lycopene, $\beta$-cryptoxanthin, lutein, and zeaxanthin, and $70.7 \%$ and $89.9 \%$ for $\gamma$-tocopherol and $\alpha$-tocopherol.

\subsection{Statistical Analysis}

The database was established by using Epi Data version 3.0, and a double data entry was carried out. For the information of demographic characteristics, the data were presented as count with percentage for categorical data, and median with interquartile range for continuous data with non-normal distribution. Before the progress of data analysis, the Shapiro-Wilk test was used to determine whether carotenoids and tocopherols in breast milk, and vitamin intake had a normal distribution or not. Due to non-normal distributions, median values (interquartile range) were performed and $\ln$ transformations were applied when doing covariance analysis, multivariate analysis, 
and correlation analysis. Differences in breast milk vitamins were compared between stages of lactating periods (0-4 days, 5-11 days, 12-30 days, 31-60 days, 61-120 days, and 121-240 days postpartum) and cities (Beijing, Suzhou, and Guangzhou cities) by using a nonparametric Kruskal-Wallis test; then, nonparametric Mann-Whitney U tests were employed to detect specific differences between the abovementioned groups further. According to the demographic characteristics of lactating women and their offspring, comparisons in carotenoids and tocopherols concentration were carried out by using covariance analysis models adjusted according to stages of lactation and research cities. Furthermore, multivariate linear regression models were explored to research the demographic influencing factors of carotenoids and tocopherols concentrations in breast milk. To research the correlations between these contents in breast milk and dietary vitamins intake, partial correlation adjusted with stages of lactation and research cities were performed. All of the analyses were carried out using the Statistical Package for the Social Sciences (SPSS Inc., Chicago, IL, USA) version 20.0, and all tests were two-tailed, with statistical significance set at $p<0.05$.

\section{Results}

The socio-economic characteristics of the lactating women are summarized in Table 1 . The mean age of the lactating women was $27.4 \pm 4.0$ years. The majority of lactating women were unemployed, had completed high school, and had a monthly household income representative of urban China. Although the majority of women had a normal BMI at present, 44\% of them had excessive GWG, and up to $48 \%$ lactating women had a cesarean delivery. Based on the stage of lactating period, there were no significant differences in the socio-economic characteristics of the lactating women such as age, offspring gender, family's per capita income, current BMI, GWG, and pregnancy duration. However, it was found that less lactating women during 121-240 days postpartum had college education or higher when compared with the others $(p<0.05)$. Meanwhile, more lactating women during $0-4$ days and 31-60 days postpartum underwent cesarean delivery when compared with those women during 5-11 days, 61-120 days, and 121-240 days postpartum $(p<0.05)$, and more lactating women during 31-60 days, $61-120$ days, and 121-240 days postpartum received dietary supplements than those women during $0-4$ days postpartum $(p<0.05)$.

Table 1. Demographic characteristics of lactating mothers according to different lactating stages.

\begin{tabular}{|c|c|c|c|c|c|c|c|}
\hline & $\begin{array}{c}\text { 0-4 Days } \\
(n=77)\end{array}$ & $\begin{array}{l}\text { 5-11 Days } \\
(n=89)\end{array}$ & $\begin{array}{c}\text { 12-30 Days } \\
(n=73)\end{array}$ & $\begin{array}{c}\text { 31-60 Days } \\
\quad(n=90)\end{array}$ & $\begin{array}{c}\text { 61-120 Days } \\
(n=90)\end{array}$ & $\begin{array}{c}\text { 121-240 Days } \\
(n=90)\end{array}$ & $p$-Value \\
\hline Age, years ${ }^{1}$ & & & & & & & 0.097 \\
\hline$<25$ & $22(28.6)$ & $27(30.3)$ & $26(35.6)$ & $18(20.0)$ & $26(28.9)$ & $36(40.0)$ & \\
\hline $25-30$ & $35(45.5)$ & $41(46.1)$ & $29(39.7)$ & $44(48.9)$ & $50(55.6)$ & $39(43.3)$ & \\
\hline$>30$ & $20(26.0)$ & $21(23.6)$ & $18(24.7)$ & $28(31.1)$ & $14(15.6)$ & $15(16.7)$ & \\
\hline Offspring gender ${ }^{1}$ & & & & & & & 0.158 \\
\hline Male & $35(45.5)$ & $51(57.3)$ & $39(53.4)$ & $48(53.3)$ & $54(60.0)$ & $43(47.8)$ & \\
\hline Female & $42(54.5)$ & $38(42.7)$ & $31(42.5)$ & $39(43.3)$ & $36(40.0)$ & $44(48.9)$ & \\
\hline Education ${ }^{1}$ & & & & & & & $0.003 *$ \\
\hline Middle school or below & $17(22.1)$ & $12(13.5)$ & $16(21.9)$ & $26(28.9)$ & $22(24.4)$ & $39(43.3)$ & \\
\hline High school & $23(29.9)$ & $31(34.8)$ & $27(37.0)$ & $22(24.4)$ & $25(27.8)$ & $23(25.6)$ & \\
\hline College or above & $36(46.8)$ & $45(50.6)$ & $29(39.7)$ & $42(46.7)$ & $41(45.6)$ & $26(28.9)$ & \\
\hline \multicolumn{4}{|c|}{ Family's per capita income, Yuan $/$ month $^{1}$} & & & & 0.140 \\
\hline$<2000$ & $16(20.8)$ & $19(21.3)$ & $16(21.9)$ & $24(26.7)$ & $26(28.9)$ & $31(34.4)$ & \\
\hline $2000-4000$ & $30(39.0)$ & 37 (41.6) & $34(46.6)$ & $41(45.6)$ & $40(44.4)$ & $41(45.6)$ & \\
\hline$>4000$ & $27(35.1)$ & $30(33.7)$ & $17(23.3)$ & $23(25.6)$ & $22(24.4)$ & $18(20.0)$ & \\
\hline Unclear & $4(5.2)$ & $3(3.4)$ & $6(8.2)$ & $2(2.2)$ & $2(2.2)$ & $0(0.0)$ & \\
\hline Delivery mode $^{1}$ & & & & & & & $0.002 *$ \\
\hline Vaginal delivery & $29(37.7)$ & $50(56.2)$ & $35(47.9)$ & $37(41.1)$ & $55(61.1)$ & $55(61.1)$ & \\
\hline Cesarean delivery & $48(62.3)$ & $37(41.6)$ & $38(52.1)$ & $53(58.9)$ & $35(38.9)$ & $34(37.8)$ & \\
\hline Present BMI ${ }^{1}$ & & & & & & & 0.075 \\
\hline Underweight & $1(1.3)$ & $5(5.6)$ & $2(2.7)$ & $2(2.2)$ & $4(4.4)$ & $8(8.9)$ & \\
\hline Normal & $48(62.3)$ & $54(60.7)$ & $47(64.4)$ & $57(63.3)$ & $69(76.7)$ & $65(72.2)$ & \\
\hline Overweight & $24(31.2)$ & $26(29.2)$ & $23(31.5)$ & $26(28.9)$ & $16(17.8)$ & $16(17.8)$ & \\
\hline Obesity & $4(5.2)$ & $3(3.4)$ & $1(1.4)$ & $5(5.6)$ & $1(1.1)$ & $1(1.1)$ & \\
\hline
\end{tabular}


Table 1. Cont.

\begin{tabular}{|c|c|c|c|c|c|c|c|}
\hline & $\begin{array}{c}\text { 0-4 Days } \\
(n=77)\end{array}$ & $\begin{array}{l}\text { 5-11 Days } \\
(n=89)\end{array}$ & $\begin{array}{c}\text { 12-30 Days } \\
(n=73)\end{array}$ & $\begin{array}{c}\text { 31-60 Days } \\
(n=90)\end{array}$ & $\begin{array}{c}\text { 61-120 Days } \\
(n=90)\end{array}$ & $\begin{array}{l}\text { 121-240 Days } \\
(n=90)\end{array}$ & $p$-Value \\
\hline Gestational weight gain ${ }^{1}$ & & & & & & & 0.300 \\
\hline Inadequate & $17(22.1)$ & $11(12.4)$ & $14(19.2)$ & $17(18.9)$ & $19(21.1)$ & $26(28.9)$ & \\
\hline Adequate & $27(35.1)$ & $29(32.6)$ & $28(38.4)$ & $32(35.6)$ & $36(40.0)$ & $25(27.8)$ & \\
\hline Excessive & $33(42.9)$ & $48(53.9)$ & $29(39.7)$ & $41(45.6)$ & $34(37.8)$ & $39(43.3)$ & \\
\hline Dietary supplements intake ${ }^{1}$ & & & & & & & 0.028 * \\
\hline Yes & $5(6.5)$ & $13(14.6)$ & $17(23.3)$ & $17(18.9)$ & $22(24.4)$ & $13(14.4)$ & \\
\hline No & $72(93.5)$ & $76(85.4)$ & $56(76.7)$ & $73(81.1)$ & $68(75.6)$ & $77(85.6)$ & \\
\hline Pregnancy duration, weeks ${ }^{2}$ & $39(38-40)$ & $39(39-40)$ & $39(38-40)$ & $39(38-40)$ & $39.5(39-40)$ & $40(39-40)$ & 0.332 \\
\hline
\end{tabular}

BMI, body mass index, was calculated as body weight by height squared $\left(\mathrm{kg} / \mathrm{m}^{2}\right)$. Data are expressed as medians (interquartile ranges) for continuous variables and count (percentage) for categorical variables. * Indicates a significant difference among six stages of lactating period $(p<0.05) .{ }^{1}$ Compared by Kruskal-Wallis test; ${ }^{2}$ Compared by chi-square test.

The concentrations of the different compounds studied at different periods of lactation are shown in Table 2. As expected, significant differences according to the different periods of lactation were observed for many compounds $(p<0.001)$. The concentrations of most of them (except for lutein, zeaxanthin, and $\gamma$-tocopherol) in milk from lactating women during $0-4$ days postpartum were significantly higher compared with those during other periods $(p<0.01)$; a decrease was observed with the advancement of lactation until it reached stable levels from 12 days postpartum. Furthermore, lutein concentrations in milk from lactating women during 0-4 days and 5-11 days postpartum were significantly higher compared with those during other periods $(p<0.01)$, while zeaxanthin and $\gamma$-tocopherol concentrations remained stable over time.

Table 2. Carotenoids and tocopherols concentrations in human milk at different lactation stages $(\mu \mathrm{g} / 100 \mathrm{~mL})$.

\begin{tabular}{|c|c|c|c|c|c|c|c|c|}
\hline & $\begin{array}{c}\text { 0-4 Days } \\
(n=77)\end{array}$ & $\begin{array}{c}\text { 5-11 Days } \\
(n=89)\end{array}$ & $\begin{array}{c}\text { 12-30 Days } \\
(n=73)\end{array}$ & $\begin{array}{c}\text { 31-60 Days } \\
(n=90)\end{array}$ & $\begin{array}{c}\text { 61-120 Days } \\
(n=90)\end{array}$ & $\begin{array}{c}\text { 121-240 Days } \\
(n=90)\end{array}$ & $p$-Value ${ }^{1}$ & Post hoc Test $^{2}$ \\
\hline$\beta$-carotene & $8.0(4.7-15.2)$ & $2.8(2.0-4.4)$ & $2.1(1.4-3.1)$ & $1.7(1.3-3.0)$ & $1.9(1.4-2.7)$ & $1.8(1.4-2.6)$ & $<0.001 *$ & $\begin{array}{c}\mathrm{P} 1>\mathrm{P} 2>\mathrm{P} 3=\mathrm{P} 4 \\
=\mathrm{P} 5=\mathrm{P} 6\end{array}$ \\
\hline$\beta$-cryptoxanthin & $6.2(2.4-12.9)$ & $3.4(1.7-5.7)$ & $2.4(1.1-3.9)$ & $1.7(1.1-2.6)$ & $1.8(1.0-4.0)$ & $2.1(1.1-3.7)$ & $<0.001$ * & $\begin{array}{c}\mathrm{P} 1>\mathrm{P} 2>\mathrm{P} 3=\mathrm{P} 4 \\
=\mathrm{P} 5=\mathrm{P} 6\end{array}$ \\
\hline Lutein & $5.7(2.9-10.2)$ & $7.0(4.6-10.3)$ & $2.2(1.2-6.3)$ & $2.9(0.9-5.9)$ & $2.8(1.2-6.5)$ & $3.7(2.4-5.9)$ & $<0.001$ * & $\begin{array}{c}\mathrm{P} 1=\mathrm{P} 2>\mathrm{P} 3=\mathrm{P} 4= \\
\mathrm{P} 5=\mathrm{P} 6\end{array}$ \\
\hline Lycopene & $6.3(4.0-9.9)$ & $2.5(1.7-4.3)$ & $1.8(1.2-2.6)$ & $1.4(1.1-2.0)$ & $1.4(1.0-2.0)$ & $1.5(1.3-2.0)$ & $<0.001$ * & $\begin{array}{c}\mathrm{P} 1>\mathrm{P} 2>\mathrm{P} 3=\mathrm{P} 4 \\
=\mathrm{P} 5=\mathrm{P} 6\end{array}$ \\
\hline Zeaxanthin & $1.0(0.6-1.5)$ & $1.4(1.0-2.2)$ & $0.8(0.4-1.5)$ & $0.8(0.4-1.4)$ & $1.0(0.4-1.4)$ & $1.1(0.8-1.4)$ & $<0.001$ * & $\begin{array}{c}\mathrm{P} 2>\mathrm{P} 1=\mathrm{P} 3=\mathrm{P} 4 \\
=\mathrm{P} 5=\mathrm{P} 6\end{array}$ \\
\hline$\alpha$-tocopherol & 645 (388-1176) & $382(236-551)$ & $239(145-396)$ & $206(126-345)$ & $212(112-300)$ & $211(135-326)$ & $<0.001 *$ & $\begin{array}{c}\mathrm{P} 1>\mathrm{P} 2>\mathrm{P} 3=\mathrm{P} 4 \\
=\mathrm{P} 5=\mathrm{P} 6\end{array}$ \\
\hline$\gamma$-tocopherol & $68(48-121)$ & $63(43-103)$ & $70(39-104)$ & $73(41-120)$ & $68(39-112)$ & $88(56-137)$ & $<0.033$ * & $\begin{array}{c}\mathrm{P} 2=\mathrm{P} 3<\mathrm{P} 6 \\
\mathrm{P} 1=\mathrm{P} 4=\mathrm{P} 5=\mathrm{P} 6\end{array}$ \\
\hline
\end{tabular}

Data are obtained from all three cities and presented as the medians (interquartile ranges). ${ }^{*}$ Indicates a significant difference among the six periods $(p<0.05) .{ }^{1}$ Compared by Kruskal-Wallis test; ${ }^{2}$ Compared by Mann-Whitney $\mathrm{U}$ test with adjusted alpha value $\left(\alpha^{\prime}=0.01\right)$. P1: 0-4 days postpartum; P2: 5-11 days postpartum; P3: 12-30 days postpartum; P4: 31-60 days postpartum; P5: 61-120 days postpartum; P6: 121-240 days postpartum.

Carotenoids and tocopherol concentrations in breast milk from lactating women in the three cities are shown in Table 3. The lycopene content in milk from Guangzhou city was significantly higher compared with those from Beijing and Suzhou cities $(p<0.001)$. Similarly, the majority of carotenoids ( $\beta$-carotene, $\beta$-cryptoxanthin, lutein, and zeaxanthin) and $\alpha$-tocopherol concentrations in milk from Beijing were significantly lower than those from Suzhou and Guangzhou $(p<0.01)$. Meanwhile, $\gamma$-tocopherol concentrations in mothers from Suzhou were the highest among the three cities $(p<0.001)$. 
Table 3. Carotenoids and tocopherols concentration of human milk from different cities (Beijing, Suzhou, and Guangzhou cities) ( $\mu \mathrm{g} / 100 \mathrm{~mL})$.

\begin{tabular}{cccccc}
\hline & $\begin{array}{c}\text { Beijing } \\
(\boldsymbol{n}=\mathbf{1 5 1})\end{array}$ & $\begin{array}{c}\text { Suzhou } \\
(\boldsymbol{n}=\mathbf{1 8 0})\end{array}$ & $\begin{array}{c}\text { Guangzhou } \\
(\boldsymbol{n}=\mathbf{1 7 8})\end{array}$ & $\boldsymbol{p}$-Value ${ }^{\mathbf{1}}$ & Post hoc Test $^{\mathbf{2}}$ \\
\hline B-carotene & $1.7(1.3-3.2)$ & $2.4(1.7-4.3)$ & $2.7(1.7-5.0)$ & $<0.001^{*}$ & $\mathrm{C} 1<\mathrm{C} 2=\mathrm{C} 3$ \\
$\beta$-cryptoxanthin & $1.1(0.8-2.0)$ & $3.6(2.1-7.7)$ & $2.8(1.7-5.2)$ & $<0.001^{*}$ & $\mathrm{C} 1<\mathrm{C} 3<\mathrm{C} 2$ \\
Lutein & $2.2(1.0-4.1)$ & $4.9(2.6-7.9)$ & $5.8(2.9-8.7)$ & $<0.001^{*}$ & $\mathrm{C} 1<\mathrm{C} 2=\mathrm{C} 3$ \\
Lycopene & $1.7(1.3-2.8)$ & $1.7(1.3-2.7)$ & $2.1(1.4-3.8)$ & $0.006^{*}$ & $\mathrm{C} 1=\mathrm{C} 2<\mathrm{C} 3$ \\
Zeaxanthin & $0.8(0.4-1.4)$ & $1.1(0.7-2.0)$ & $1.1(0.7-1.5)$ & $<0.001^{*}$ & $\mathrm{C} 1<\mathrm{C} 2=\mathrm{C} 3$ \\
$\alpha$-tocopherol & $215(117-333)$ & $296(208-478)$ & $285(148-479)$ & $<0.001^{*}$ & $\mathrm{C} 1<\mathrm{C} 2=\mathrm{C} 3$ \\
$\gamma$-tocopherol & $71(48-107)$ & $94(59-148)$ & $53(31-88)$ & $<0.001^{*}$ & $\mathrm{C} 3<\mathrm{C} 1<\mathrm{C} 2$ \\
\hline
\end{tabular}

Data are obtained from all six lactation stages and presented as the medians (interquartile ranges). ${ }^{*}$ Indicates a significant difference among the three cities $(p<0.05) .{ }^{1}$ Compared by Kruskal-Wallis test; ${ }^{2}$ Compared by Mann-Whitney U test with adjusted alpha value $\left(\alpha^{\prime}=0.01\right)$. C1: Beijing; C2: Suzhou; C3: Guangzhou.

Comparisons of the concentrations by characteristics of lactating women and their offspring are provided in Table 4 and supplementary material Table S1. There were no significant associations detected between maternal age, offspring gender, household income, maternal GWG, dietary supplement intake, and tocopherols in breast milk $(p>0.05)$. However, zeaxanthin concentrations in lactating women with vaginal delivery were significant higher compared with those with cesarean delivery $(p<0.05)$. In addition, zeaxanthin concentrations in women with a college education level or above were significant lower than in women with a middle school education level or below $(p<0.01)$. Besides, associations were found between $\beta$-carotene and zeaxanthin concentrations and maternal BMI, which indicated that $\beta$-carotene concentrations in lactating women with normal BMI were higher than those in overweight women, while zeaxanthin concentrations in milk from underweight lactating women were higher than those from mothers with a normal BMI.

Table 4. Comparisons of the carotenoids concentration in human milk by the characteristics of lactating women.

\begin{tabular}{|c|c|c|c|c|c|c|c|c|}
\hline & \multicolumn{2}{|l|}{$\beta$-carotene } & \multicolumn{2}{|c|}{$\beta$-cryptoxanthin } & \multicolumn{2}{|l|}{ Lutein } & \multicolumn{2}{|l|}{ Zeaxanthin } \\
\hline & $\begin{array}{c}\text { Adjusted }^{1} \beta \\
(95 \% \mathrm{CI})\end{array}$ & SEM & $\begin{array}{l}\text { Adjusted }^{1} \beta \\
(95 \% \mathrm{CI})\end{array}$ & SEM & $\begin{array}{c}\text { Adjusted }^{1} \beta \\
(95 \% \mathrm{CI})\end{array}$ & SEM & Adjusted $^{1} \beta(95 \% \mathrm{CI})$ & SEM \\
\hline \multicolumn{9}{|l|}{ Age, years } \\
\hline$<25$ & $-0.05(-0.18,0.08)$ & 0.07 & $0.11(-0.06,0.28)$ & 0.09 & $-0.13(-0.35,0.08)$ & 0.11 & $-0.01(-0.14,0.15)$ & 0.07 \\
\hline $25-30$ & Reference & & Reference & & Reference & & Reference & \\
\hline$>30$ & $0.10(-0.03,0.23)$ & 0.07 & $0.11(-0.07,0.29)$ & 0.09 & $-0.03(-0.15,0.19)$ & 0.11 & $0.12(-0.03,0.27)$ & 0.08 \\
\hline \multicolumn{9}{|l|}{ Education } \\
\hline Middle school or below & Reference & & Reference & & Reference & & Reference & \\
\hline High school & $0.03(-0.11,0.17)$ & 0.07 & $-0.18(-0.36,0.01)$ & 0.09 & $0.12(-0.11,0.35)$ & 0.12 & $-0.14(-0.30,0.02)$ & 0.08 \\
\hline College or above & $0.09(-0.04,0.23)$ & 0.07 & $-0.12(-0.30,0.06)$ & 0.09 & $0.08(-0.14,0.31)$ & 0.11 & $-0.15(-0.31,-0.00)$ * & 0.08 \\
\hline Delivery mode & & & & & 0 & & & \\
\hline Vaginal delivery & $0.04(-0.07,0.15)$ & 0.05 & $0.03(-0.11,0.17)$ & 0.07 & $0.14(-0.04,0.31)$ & 0.09 & $0.13(0.02,0.25) *$ & 0.06 \\
\hline Cesarean delivery & Reference & & Reference & & Reference & & Reference & \\
\hline Current BMI & & & & & 0 & & & \\
\hline $\begin{array}{l}\text { Underweight } \\
\text { Normal }\end{array}$ & $\begin{array}{c}0.01(-0.25,0.26) \\
\text { Reference }\end{array}$ & 0.13 & $\begin{array}{c}-0.02(-0.35,0.32) \\
\text { Reference }\end{array}$ & 0.17 & $0.32(-0.09,0.74)$ & 0.21 & $\begin{array}{c}0.29(0.01,0.57) * \\
\text { Reference }\end{array}$ & 0.14 \\
\hline Overweight & $-0.17(-0.29,-0.05) *$ & 0.06 & $-0.16(-0.32,0.00)$ & 0.08 & $-0.11(-0.31,0.09)$ & 0.10 & $-0.07(-0.21,0.07)$ & 0.07 \\
\hline Obesity & $-0.24(-0.54,0.07)$ & 0.16 & $-0.16(-0.57,0.24)$ & 0.21 & $0.16(-0.34,0.66)$ & 0.26 & $-0.18(-0.52,0.16)$ & 0.17 \\
\hline
\end{tabular}

CI, confidence interval; SEM, standard error of mean. Multivariate linear regression model considering carotenoids in breast milk as the dependent variable and the other variables studies as independent variables. ${ }^{1}$ Adjusted for periods of lactation (0-4 days, 5-11 days, 12-30 days, 31-60 days, 61-120 days, and 121-240 days postpartum), cities (Beijing, Suzhou, and Guangzhou cities), and other independent influencing factors listed above. * Indicates a significant difference when compared with the reference $(p<0.05)$. $\beta$-carotene: $\mathrm{R}^{2}=0.482, p<0.001 ; \beta$-cryptoxanthin: $\mathrm{R}^{2}=0.366, p<0.001 ;$ Lutein: $\mathrm{R}^{2}=0.282, p<0.001$; Zeaxanthin: $\mathrm{R}^{2}=0.124, p<0.001$

The results from a 24-h food intake recall showed that dietary vitamin A and total carotenoids were not associated with all of the carotenoids in breast milk when adjusted with different cities and lactation period $(p>0.05)$ (Table 5). Similarly, no significant associations were found between vitamin E and $\alpha$-tocopherol intake, and $\alpha$ - and $\gamma$-tocopherol in human milk $(p>0.05)$. 
Table 5. The associations between vitamins intake and concentrations of carotenoids and tocopherols in breast milk.

\begin{tabular}{ccccccccc}
\hline & & $\beta$-carotene & $\beta$-cryptoxanthin & Lutein & Lycopene & Zeaxanthin & $\alpha$-tocopherol & $\gamma$-tocopherol \\
\hline Dietary intake of vitamin A & $R$ & 0.022 & 0.026 & 0.027 & -0.007 & 0.075 & - & - \\
Dietary intake of total carotenoids & $p^{1}$ & 0.618 & 0.562 & 0.537 & 0.881 & 0.093 & - & - \\
& $R$ & 0.055 & 0.002 & 0.007 & -0.038 & 0.003 & - & - \\
Dietary intake of vitamin E & $p^{1}$ & 0.220 & 0.963 & 0.880 & 0.398 & 0.948 & - & - \\
Dietary intake of $\alpha$-tocopherol & $R$ & - & - & - & - & - & -0.083 & 0.006 \\
& $p^{1}$ & - & - & - & - & - & 0.063 & 0.885 \\
& $R$ & - & - & - & - & - & -0.033 & -0.084 \\
\hline
\end{tabular}

1 Partial correlation was performed to analyze the correlations adjusted with cities (Beijing, Suzhou, and Guangzhou cities) and periods of lactating (0-4 days, 5-11 days, 12-30 days, 31-60 days, 61-120 days, and 121-240 days emphpostpartum).

\section{Discussion}

According to the literature $[26,35,48,49]$, colostrum is the first milk secretion after delivery, persisting until the seventh or 10th day postpartum; it is followed by the secretion of transitional milk from around the eight and up to 15th day postpartum; from then, mature milk is secreted, which shows a relative stable composition. Considering that compositions in human milk do not change abruptly, and the timing varies from one mother to another, there are no clear boundaries among colostrum, transitional milk, and mature milk [50-52]. Therefore, we collected human milk during consecutive lactation stages including 0-4 days, 5-11 days, 12-30 days, 31-60 days, 61-120 days, and 121-240 days postpartum to characterize carotenoids and tocopherols in human milk along lactation. In the present study, due to the nature of its design, it was difficult to exactly classify the human milk collected within 5-11 days and 12-30 days postpartum as either colostrum or transitional milk. On the contrary, human milk collected within 0-4 days and 31-240 days postpartum was clearly classified as colostrum and mature milk, respectively.

The highest concentrations of carotenoids and tocopherols were found in colostrum, after that, the concentrations of the different compounds observed in milk at 12-30 days postpartum were close to those collected in 30-240 days postpartum, which may mean that the changes on carotenoids and tocopherols milk composition gradually slow down until reaching a relatively stable level after 12 days postpartum. In accordance with previous studies $[26,48,49,53]$, the levels of most of the compounds ( $\beta$-carotene, $\beta$-cryptoxanthin, lutein, lycopene, and $\alpha$-tocopherol), except for zeaxanthin and $\gamma$-tocopherol, decreased along with lactating stage. The evolution trend of $\gamma$-tocopherol concentrations in our study were generally comparable with those in Japan [53] $(0.111 \pm 0.048 \mathrm{mg} / 100 \mathrm{~mL}$ in 6-10 days postpartum; $0.155 \pm 0.126 \mathrm{mg} / 100 \mathrm{~mL}$ in $11-20$ days postpartum, $0.105 \pm 0.059 \mathrm{mg} / 100 \mathrm{~mL}$ in 21-89 days postpartum; $0.120 \pm 0.046 \mathrm{mg} / 100 \mathrm{~mL}$ in $90-180$ days postpartum; $0.086 \pm 0.043 \mathrm{mg} / 100 \mathrm{~mL}$ in 6-10 days postpartum).

The carotenoid content in human milk has been studied in several multinational studies, but very few studies report data for a large lactation period (0-240 days postpartum). Canfield et al. [21] assessed the levels of carotenoids in human milk from nine countries (five in Asia or the Pacific Rim, three in the Americas, and one in Europe), and found that the concentrations varied greatly among the countries, with only moderate disparities in $\beta$-carotene. Regional variability was also found in the longitudinal study of Lipkie et al. [23]. In the present study, we found the median $\beta$-carotene concentration in mature milk to be in the same range as that found in Australian, Canadian, Chilean, Japanese, Mexican, Filipinos, English, American, and German mothers in several studies [21,25,26,54]; while the median $\beta$-carotene concentrations in colostrum milk were lower than those reported in Germany [25] or Japan [54]. Regarding the relative distribution of carotenoids in Chinese milk, lutein was found to be the major component in mature human milk, which is in agreement with data published by Lipkie et al. [23] and Canfield et al. [21]. On the contrary, $\beta$-carotene was the major carotenoid in colostrum, in agreement with a previous longitudinal study in the United States by Song et al. [55].

Differences in carotenoid content of the milk from the three different cities were also observed; milk from Beijing contained significantly lower amounts than milk from Guangzhou or Suzhou for 
most of carotenoids; while the concentration of lycopene in samples from Guangzhou was higher than the other cities. Taking into consideration that all of the samples were collected with the same protocol and analyzed by the same laboratory, it seems reasonable to believe that the main reason for this difference are environmental factors such as the lower consumption of carotenoid-rich vegetables and fruits. However, more research is needed to confirm the regional differences and elucidate their potential mechanisms.

Median $\alpha$-tocopherol concentrations $(211 \mu \mathrm{g} / 100 \mathrm{~mL})$ in Chinese mature milk were lower than those found by researchers in Germany [26], Greece [56], Turkey [36], Poland [49], Canada [57], and Japan [53]. In general, the $\alpha$-tocopherol content in Chinese milk, not only in colostrum but also in mature milk, was lower than those in industrialized countries, as well as with non-industrialized countries. The large inter-subject variation might be primarily due to dietary habits, the use of dietary supplements, food fortification, or genetic differences among different ethnicities; or to methodological factors, such as the postpartum date of collection, collection of foremilk or hindmilk, or the collection from a single breast of from both breasts.

To date, there is not much evidence of the tocopherols concentration in Chinese human milk. In our study, the median colostrum $\alpha$-tocopherol concentrations $(645 \mu \mathrm{g} / 100 \mathrm{~mL})$ were similar to those reported in different groups of Chinese lactating women [58,59] and Polish women [48], but lower than those from German [26] and Brazilian mothers [60], and higher than those of Tunisian [39], Japanese [53], and Inner Mongolia mothers in China [22]. Our results in mature milk (30-240 days postpartum) align well to published data [22,25,26,36,38,48,53,56,57]. Intra-country variability was also found, with the highest levels of tocopherols (26.8 $\mathrm{mg} \alpha$-tocopherol) found in mothers from Suzhou. Environmental factors such as dietary intake are likely the reason for this. The intake of $4 \mathrm{mg} / \mathrm{day}$ of vitamin $\mathrm{E}$ ( $\alpha$-tocopherol) for infants aged less than six months is recommended based on a value of adequate intake (AI) in the Dietary Reference Intakes (DRIs) [61]. According to the recommendations of the Institute of Medicine (IOM) [62], we estimated the supply of vitamin E for infants in the present study using the average quantity of milk consumed by infants aged 0-6 $\mathrm{m}(780 \mathrm{~mL})$, and found that the possible effect of these milks offered to the infants was 5.0, 3.0, 1.9, 1.6, 1.7, $1.6 \mathrm{mg} /$ day of vitamin E, respectively. Our findings suggested that only colostrum reached an AI proposal for the infant aged less than six months; therefore, the implementation of procedures to increase vitamin E concentrations in milk would be important.

Some nutritional, obstetric, and socio-economic factors have been implicated as being associated with the vitamin A and E concentrations in milk $[36,39,60]$. In our study, there were no associations detected between the contents of $\beta$-cryptoxanthin, lutein, lycopene, $\alpha$-tocopherol, and $\gamma$-tocopherol, and the socio-economic characteristics of women and their offspring, such as maternal age, household income, maternal GWG, or supplement intake. This data provides evidence to suggest that the concentrations of these compounds in breast milk are independent of socio-economic conditions and nutrition in pregnancy. On the contrary, we found associations between the human milk $\beta$-carotene and zeaxanthin concentrations, and current BMI. These inverse correlations between some of the carotenoids and current BMI may be due to the underlying mechanism that an excess of body fat increases the consumption of all antioxidant elements in the diet [63], so that lactating women with a higher BMI and more body fat consumed more vitamins A and E than those with lower BMI, resulting in lower carotenoids in human milk. Our results were similar with the previous finding [37] that the percentage of body fat in the lactating women was negatively associated to the concentration of vitamin A in breast milk. In addition, maternal education presented an inverse relationship with lower median concentrations of zeaxanthin among women with high levels of education. Mothers undergoing cesarean delivery presented lower zeaxanthin levels in human milk. Previous studies have associated cesarean delivery with lower colostrum protein content [64] and decreased oxidative stress in colostrum [65], suggesting that cesarean delivery may be detrimental for human milk. Considering that carotenoids contribute to the total anti-oxidative effect of human milk, this relationship of carotenoids 
and delivery mode requires further investigation to elucidate the possible causal pathways of these mechanisms including organismal regulation, nutrition, and environment.

Previous studies $[36,37,66]$ suggested vitamins A and E in human milk were associated with maternal stores, dietary supplements, fortified foods, and dietary intake. However, our results indicated that neither vitamin A nor E intakes from one 24-h dietary recall was associated with vitamin A and $\mathrm{E}$ in human milk, which may be due to the inherent intake variability of one single 24-h dietary recall questionnaire, which did not allow for estimations of an individual's usual diet, and therefore, it is likely to under- or overestimate some nutrient intakes. Moreover, Jiang et al. [67] found no significant correlation between dietary constituents and $\alpha$-tocopherol, which is in line with our findings.

There were some limitations to the present study. Firstly, nutrient intake determined by one 24-h recall may introduce some bias by under- or overestimating long-term dietary habits. This variability may result in difficulties accurately estimating individual's intake when compared with three days dietary recall. Secondly, little is known about the levels of vitamins A and E in maternal plasma associated with the corresponding milk, which would be better than dietary intake to assess maternal nutrient status, due to the impossibility to collect such samples in our research. Thirdly, since our design was a cross-sectional study, we could not collect direct evidence about changes in vitamins A and $\mathrm{E}$ with lactation stages. These points should be addressed in future studies. Fourthly, although strict inclusion criteria and exclusion criteria were implemented, we could not ensure that the milk in our study population was sufficient to provide the needed amounts of these compounds. Indeed, our results showed that all of these milks, except for colostrum, did not meet an infant's nutritional requirement of vitamin E. However, we could not estimate the possible supply of vitamin A to the infant in the present study, due to the lacking detection of retinol in human milk.

\section{Conclusions}

The total concentrations of carotenoids ( $\beta$-carotene and $\beta$-cryptoxanthin, lutein, lycopene, and zeaxanthin) and vitamin E (as $\alpha$ - and $\gamma$-tocopherol) were studied in human milk from healthy Chinese women. In summary, our results agreed with previous studies, and suggested that the stage of lactation, regional differences, and obstetric and socio-economic factors might have an effect on the human milk concentrations of carotenoids and tocopherol in healthy Chinese mothers. In view of the great importance of these compounds in human milk to ensure the optimal growth and development of infants, research should continue to explore the biological significance of such results, and improve knowledge on the unique composition of human milk.

Supplementary Materials: The following are available online at www.mdpi.com/2072-6643/9/11/1229/s1, Table S1: Comparisons of carotenoids and tocopherols concentration according to characteristics of lactating women and their offspring $(\mu \mathrm{g} / 100 \mathrm{~mL})$.

Acknowledgments: The authors would like to thank the participants who volunteered for this study, Lawrence Li for project support and guidance, Celia Ning for project management, Qiaoji Li for clinical project management, Yindong Zheng and Carlos Antonio de Castro for statistical analysis advice and Emilie Ba for data management. Special acknowledgment to Ai Zhao and the project staff at Peking University School of Public Health, to Jiaji Wang at Guangzhou University School of Public Health and Liqiang Qin at Soochow University School of Public Health for recruitment and data collection. Nestlé Research Center and Nestlé Nutrition Institute China sponsored the study. The views and opinions expressed in this manuscript are those of the authors and do not necessarily reflect the opinions and recommendations of Nestlé.

Author Contributions: Yong Xue and Esther Campos-Giménez interpreted the results, drafted, reviewed and revised the initial manuscript. Karine Meisser Redeuil, Antoine Lévèques and Lucas Actis-Goretta contributed to analysis of samples. Gerard Vinyes-Pares contributed to the study design, drafted, and reviewed the initial manuscript. Yong Xue contributed to statistical design. Yumei Zhang and Peiyu Wang contributed to study design and field collection. Sagar K. Thakkar contributed to the study design, breast milk sampling protocol, interpretation of the results. All authors read and approved the final manuscript.

Conflicts of Interest: The authors declare no conflict of interest. The founding sponsors had no role in the design of the study; in the collection, analyses, or interpretation of data; in the writing of the manuscript, and in the decision to publish the results. 


\section{References}

1. World Health Organization/United Nations Children's Fund. Global Strategy for Infant and Young Child Feeding; World Health Organization: Geneva, Switzerland, 2003.

2. Onyango, A.W.; Receveur, O.; Esrey, S.A. The contribution of breast milk to toddler diets in western Kenya. Bull. World Health Organ. 2002, 80, 292-299. [PubMed]

3. Allen, L.H.; Haskell, M. Vitamin A requirements of infants under six months of age. Food Nutr. Bull. 2001, 22, 214-234. [CrossRef]

4. World Health Organization and Food and Agriculture Organization. Vitamin and Mineral Requirements in Human Nutrition, 2nd ed.; World Health Organization: Geneva, Switzerland, 2004.

5. Stam, J.; Sauer, P.J.; Boehm, G. Can we define an infant's need from the composition of human milk? Am. J. Clin. Nutr. 2013, 98, S521-S528. [CrossRef] [PubMed]

6. Powers, H.J. Vitamin requirements for term infants: Considerations for infant formulae. Nutr. Res. Rev. 1997, 10, 1-33. [CrossRef] [PubMed]

7. Ball, G.F.M. Vitamins: Their Role in the Human Body; Ball, G.F.M., Ed.; Blackwell Science Ltd.: Hoboken, NJ, USA, 2004; Chapter 7; pp. 133-187.

8. Ball, G.F.M. Vitamins: Their Role in the Human Body; Ball, G.F.M., Ed.; Blackwell Science Ltd.: Hoboken, NJ, USA, 2004; Chapter 9; pp. 234-255.

9. Allen, L.H. Multiple micronutrients in pregnancy and lactation: An overview. Am. J. Clin. Nutr. 2005, 81, S1206-S1212.

10. Picciano, M.F. Pregnancy and lactation: Physiological adjustments, nutritional requirements and the role of dietary supplements. J. Nutr. 2003, 133, S1997-S2002.

11. Qian, J.; Chen, T.; Lu, W.; Wu, S.; Zhu, J. Breast milk macro- and micronutrient composition in lactating mothers from suburban and urban Shanghai. J. Paediatr. Child Health 2010, 46, 115-120. [CrossRef] [PubMed]

12. Yang, T.; Zhang, Y.; Ning, Y.; You, L.; Ma, D.; Zheng, Y.; Yang, X.; Li, W.; Wang, J.; Wang, P. Breast milk macronutrient composition and the associated factors in urban Chinese mothers. Chin. Med. J. 2014, 127, 1721-1725. [PubMed]

13. Ding, M.; Li, W.; Zhang, Y.; Wang, X.; Zhao, A.; Zhao, X.; Wang, P.; Sheng, Q.H. Amino acid composition of lactating mothers' milk and confinement diet in rural north China. Asia Pac. J. Clin. Nutr. 2010, 19, 344-349. [PubMed]

14. Xiang, M.; Harbige, L.S.; Zetterstrom, R. Breast milk levels of zinc and omega-6 polyunsaturated fatty acids and growth of healthy Chinese infants. Acta Paediatr. 2007, 96, 387-390. [CrossRef] [PubMed]

15. Peng, Y.M.; Zhang, T.Y.; Wang, Q.; Zetterstrom, R.; Strandvik, B. Fatty acid composition in breast milk and serum phospholipids of healthy term Chinese infants during first 6 weeks of life. Acta Paediatr. 2007, 96, 1640-1645. [CrossRef] [PubMed]

16. Xiang, M.; Lei, S.; Li, T.; Zetterstrom, R. Composition of long chain polyunsaturated fatty acids in human milk and growth of young infants in rural areas of northern China. Acta Paediatr. 1999, 88, 126-131. [CrossRef] [PubMed]

17. Chen, Z.Y.; Kwan, K.Y.; Tong, K.K.; Ratnayake, W.M.; Li, H.Q.; Leung, S.S. Breast milk fatty acid composition: A comparative study between Hong Kong and Chongqing Chinese. Lipids 1997, 32, 1061-1067. [CrossRef] [PubMed]

18. Wan, Z.X.; Wang, X.L.; Xu, L.; Geng, Q.; Zhang, Y. Lipid content and fatty acids composition of mature human milk in rural north China. Br. J. Nutr. 2010, 103, 913-916. [CrossRef] [PubMed]

19. Ruan, C.; Liu, X.; Man, H.; Ma, X.; Lu, G.; Duan, G.; DeFrancesco, C.A.; Connor, W.E. Milk composition in women from five different regions of China: The great diversity of milk fatty acids. J. Nutr. 1995, 125, 2993-2998. [PubMed]

20. Li, J.; Fan, Y.; Zhang, Z.; Yu, H.; An, Y.; Kramer, J.K.; Deng, Z. Evaluating the trans fatty acid, CLA, PUFA and erucic acid diversity in human milk from five regions in China. Lipids 2009, 44, 257-271. [CrossRef] [PubMed]

21. Canfield, L.M.; Clandinin, M.T.; Davies, D.P.; Fernandez, M.C.; Jackson, J.; Hawkes, J.; Goldman, W.J.; Pramuk, K.; Reyes, H.; Sablan, B.; et al. Multinational study of major breast milk carotenoids of healthy mothers. Eur. J. Nutr. 2003, 42, 133-141. [PubMed] 
22. Shi, Y.D.; Sun, G.Q.; Zhang, Z.G.; Deng, X.; Kang, X.H.; Liu, Z.D.; Ma, Y.; Sheng, Q.H. The chemical composition of human milk from Inner Mongolia of China. Food Chem. 2011, 127, 1193-1198. [CrossRef] [PubMed]

23. Lipkie, T.E.; Morrow, A.L.; Jouni, Z.E.; McMahon, R.J.; Ferruzzi, M.G. Longitudinal survey of carotenoids in human milk from urban cohorts in China, Mexico, and the USA. PLoS ONE 2015, 10, e0127729. [CrossRef] [PubMed]

24. Campos, J.M.; Paixao, J.A.; Ferraz, C. Fat-soluble vitamins in human lactation. Int. J. Vitam. Nutr. Res. 2007, 77, 303-310. [CrossRef] [PubMed]

25. Schweigert, F.J.; Bathe, K.; Chen, F.; Buscher, U.; Dudenhausen, J.W. Effect of the stage of lactation in humans on carotenoid levels in milk, blood plasma and plasma lipoprotein fractions. Eur. J. Nutr. 2004, 43, 39-44. [CrossRef] [PubMed]

26. Macias, C.; Schweigert, F.J. Changes in the concentration of carotenoids, vitamin A, alpha-tocopherol and total lipids in human milk throughout early lactation. Ann. Nutr. Metab. 2001, 45, 82-85. [CrossRef] [PubMed]

27. Gossage, C.P.; Deyhim, M.; Yamini, S.; Douglass, L.W.; Moser-Veillon, P.B. Carotenoid composition of human milk during the first month postpartum and the response to beta-carotene supplementation. Am. J. Clin. Nutr. 2002, 76, 193-197. [PubMed]

28. Nagayama, J.; Noda, K.; Uchikawa, T.; Maruyama, I.; Shimomura, H.; Miyahara, M. Effect of maternal chlorella supplementation on carotenoid concentration in breast milk at early lactation. Int. J. Food Sci. Nutr. 2014, 65, 573-576. [CrossRef] [PubMed]

29. Canfield, L.M.; Giuliano, A.R.; Neilson, E.M.; Blashil, B.M.; Graver, E.J.; Yap, H.H. Kinetics of the response of milk and serum beta-carotene to daily beta-carotene supplementation in healthy, lactating women. Am. J. Clin. Nutr. 1998, 67, 276-283. [PubMed]

30. Turner, T.; Burri, B.J.; Jamil, K.M.; Jamil, M. The effects of daily consumption of beta-cryptoxanthin-rich tangerines and beta-carotene-rich sweet potatoes on vitamin A and carotenoid concentrations in plasma and breast milk of Bangladeshi women with low vitamin a status in a randomized controlled trial. Am. J. Clin. Nutr. 2013, 98, 1200-1208. [PubMed]

31. Sherry, C.L.; Oliver, J.S.; Renzi, L.M.; Marriage, B.J. Lutein supplementation increases breast milk and plasma lutein concentrations in lactating women and infant plasma concentrations but does not affect other carotenoids. J. Nutr. 2014, 144, 1256-1263. [CrossRef] [PubMed]

32. Stuetz, W.; Carrara, V.I.; McGready, R.; Lee, S.J.; Erhardt, J.G.; Breuer, J.; Biesalski, H.K.; Nosten, F.H. Micronutrient status in lactating mothers before and after introduction of fortified flour: Cross-sectional surveys in Maela refugee camp. Eur. J. Nutr. 2012, 51, 425-434. [CrossRef] [PubMed]

33. Lietz, G.; Mulokozi, G.; Henry, J.C.; Tomkins, A.M. Xanthophyll and hydrocarbon carotenoid patterns differ in plasma and breast milk of women supplemented with red palm oil during pregnancy and lactation. J. Nutr. 2006, 136, 1821-1827. [PubMed]

34. Webb, A.L.; Aboud, S.; Furtado, J.; Murrin, C.; Campos, H.; Fawzi, W.W.; Villamor, E. Effect of vitamin supplementation on breast milk concentrations of retinol, carotenoids and tocopherols in HIV-infected Tanzanian women. Eur. J. Clin. Nutr. 2009, 63, 332-339. [CrossRef] [PubMed]

35. Lima, M.S.; Dimenstein, R.; Ribeiro, K.D. Vitamin E concentration in human milk and associated factors: A literature review. J. Pediatr. 2014, 90, 440-448. [CrossRef] [PubMed]

36. Tokusoglu, O.; Tansug, N.; Aksit, S.; Dinc, G.; Kasirga, E.; Ozcan, C. Retinol and alpha-tocopherol concentrations in breast milk of Turkish lactating mothers under different socio-economic status. Int. J. Food Sci. Nutr. 2008, 59, 166-174. [CrossRef] [PubMed]

37. Mello-Neto, J.; Rondo, P.H.; Oshiiwa, M.; Morgano, M.A.; Zacari, C.Z.; Domingues, S. The influence of maternal factors on the concentration of vitamin A in mature breast milk. Clin. Nutr. 2009, 28, 178-181. [CrossRef] [PubMed]

38. Szlagatys-Sidorkiewicz, A.; Zagierski, M.; Luczak, G.; Macur, K.; Baczek, T.; Kaminska, B. Maternal smoking does not influence vitamin A and E concentrations in mature breastmilk. Breastfeed. Med. 2012, 7, 285-289. [CrossRef] [PubMed]

39. Fares, S.; Sethom, M.M.; Kacem, S.; Ksibi, I.; Feki, M.; Jebnoun, S.; Kaabachi, N. Retinol and alpha-tocopherol in the colostrum of lactating Tunisian women delivering prematurely: Associations with maternal characteristics. Pediatr. Neonatol. 2016, 57, 120-126. [CrossRef] [PubMed] 
40. De Azeredo, V.B.; Trugo, N.M. Retinol, carotenoids, and tocopherols in the milk of lactating adolescents and relationships with plasma concentrations. Nutrition 2008, 24, 133-139. [CrossRef] [PubMed]

41. Feng, X.L.; Xu, L.; Guo, Y.; Ronsmans, C. Factors influencing rising caesarean section rates in China between 1988 and 2008. Bull. World Health Organ. 2012, 90, 30-39. [CrossRef] [PubMed]

42. Hou, L.; Li, G.; Zou, L.; Li, C.; Chen, Y.; Yuan, Y.; Wang, X.; Jia, C.; Zhang, W. Cesarean delivery rate and indications in mainland China: A cross sectional study in 2011. Zhonghua Fu Chan Ke Za Zhi 2014, 49, 728-735. [PubMed]

43. Cui, Z.; Dibley, M.J. Trends in dietary energy, fat, carbohydrate and protein intake in Chinese children and adolescents from 1991 to 2009. Br. J. Nutr. 2012, 108, 1292-1299. [CrossRef] [PubMed]

44. Rasmussen, K.M.; Yaktine, A.L. Weight Gain during Pregnancy: Reexamining the Guidelines; National Academies Press: Washington, DC, USA, 2009.

45. Yang, Y.X.; Wang, G.Y.; Pan, X.C. China Food Composition Tables (Book 1), 2nd ed.; Beijing Medical University Press: Beijing, China, 2009.

46. Yang, Y.X.; Wang, G.Y.; Pan, X.C. China Food Composition Tables 2004 (Book 2), 2nd ed.; Beijing Medical University Press: Beijing, China, 2005.

47. Agency, S.A.T. Standard Tables of Food Composition in Japan (In Japanese), 4th ed.; Ishiyaku Shuppan Publishers, Inc.: Tokyo, Japan, 2005.

48. Szlagatys-Sidorkiewicz, A.; Zagierski, M.; Jankowska, A.; Luczak, G.; Macur, K.; Baczek, T.; Korzon, M.; Krzykowski, G.; Martysiak-Zurowska, D.; Kaminska, B. Longitudinal study of vitamins A, E and lipid oxidative damage in human milk throughout lactation. Early Hum. Dev. 2012, 88, 421-424. [CrossRef] [PubMed]

49. Martysiak-Zurowska, D.; Szlagatys-Sidorkiewicz, A.; Zagierski, M. Concentrations of alpha- and gamma- tocopherols in human breast milk during the first months of lactation and in infant formulas. Matern. Child Nutr. 2013, 9, 473-482. [CrossRef] [PubMed]

50. Marcuzzi, A.; Vecchi Brumatti, L.; Caruso, L.; Copertino, M.; Davanzo, R.; Radillo, O.; Comar, M.; Monasta, L. Presence of IL-9 in paired samples of human colostrum and transitional milk. J. Hum. Lact. 2013, 29, $26-31$. [CrossRef] [PubMed]

51. Khodayar-Pardo, P.; Mira-Pascual, L.; Collado, M.C.; Martínez-Costa, C. Impact of lactation stage, gestational age and mode of delivery on breast milk microbiota. J. Perinatol. 2014, 34, 599-605. [CrossRef] [PubMed]

52. Pons, S.M.; Bargalló, A.C.; Folgoso, C.C.; López Sabater, M.C. Triacylglycerol composition in colostrum, transitional and mature human milk. Eur. J. Clin. Nutr. 2000, 54, 878-882. [CrossRef] [PubMed]

53. Sakurai, T.; Furukawa, M.; Asoh, M.; Kanno, T.; Kojima, T.; Yonekubo, A. Fat-soluble and water-soluble vitamin contents of breast milk from Japanese women. J. Nutr. Sci. Vitaminol. 2005, 51, 239-247. [CrossRef] [PubMed]

54. Kamao, M.; Tsugawa, N.; Suhara, Y.; Wada, A.; Mori, T.; Murata, K.; Nishino, R.; Ukita, T.; Uenishi, K.; Tanaka, K.; et al. Quantification of fat-soluble vitamins in human breast milk by liquid chromatography-tandem mass spectrometry. J. Chromatogr. B Anal. Technol. Biomed. Life Sci. 2007, 859, 192-200. [CrossRef] [PubMed]

55. Song, B.J.; Jouni, Z.E.; Ferruzzi, M.G. Assessment of phytochemical content in human milk during different stages of lactation. Nutrition 2013, 29, 195-202. [CrossRef] [PubMed]

56. Antonakou, A.; Chiou, A.; Andrikopoulos, N.K.; Bakoula, C.; Matalas, A.L. Breast milk tocopherol content during the first six months in exclusively breastfeeding Greek women. Eur. J. Nutr. 2011, 50, 195-202. [CrossRef] [PubMed]

57. Tijerina-Saenz, A.; Innis, S.M.; Kitts, D.D. Antioxidant capacity of human milk and its association with vitamins A and E and fatty acid composition. Acta Paediatr. 2009, 98, 1793-1798. [CrossRef] [PubMed]

58. Zheng, M.C.; Zhou, L.S.; Zhang, G.F. Alpha-tocopherol content of breast milk in China. J. Nutr. Sci. Vitaminol. 1993, 39, 517-520. [CrossRef] [PubMed]

59. Zheng, M.C.; Zhang, G.F.; Zhou, L.S.; Guo, X.G.; Quan, Y.F. Alpha-tocopherol concentrations in human milk from mothers of preterm and full-term infants in China. Biomed. Environ. Sci. 1993, 6, 259-264. [PubMed]

60. Grilo, E.C.; Lira, L.Q.; Dimenstein, R.; Ribeiro, K.D. Influence of prematurity and birth weight on the concentration of alpha-tocopherol in colostrum milk. Rev. Paul. Pediatr. 2013, 31, 473-479. [CrossRef] [PubMed] 
61. Monsen, E.R. Dietary reference intakes for the antioxidant nutrients: Vitamin C, vitamin E, selenium, and carotenoids. J. Am. Diet. Assoc. 2000, 100, 637-640. [CrossRef]

62. Institute of Medicine. Dietary Reference Intakes for Vitamin E, Selenium, and Carotenoids; National Academies Press: Washington, DC, USA, 2000.

63. Viroonudomphol, D.; Pongpaew, P.; Tungtrongchitr, R.; Changbumrung, S.; Tungtrongchitr, A.; Phonrat, B.; Vudhivai, N.; Schelp, F.P. The relationships between anthropometric measurements, serum vitamin $\mathrm{A}$ and $\mathrm{E}$ concentrations and lipid profiles in overweight and obese subjects. Asia Pac. J. Clin. Nutr. 2003, 12, 73-79. [PubMed]

64. Dizdar, E.A.; Sari, F.N.; Degirmencioglu, H.; Canpolat, F.E.; Oguz, S.S.; Uras, N.; Dilmen, U. Effect of mode of delivery on macronutrient content of breast milk. J. Matern. Fetal Neonatal Med. 2014, 27, 1099-1102. [CrossRef] [PubMed]

65. Simsek, Y.; Karabiyik, P.; Polat, K.; Duran, Z.; Polat, A. Mode of delivery changes oxidative and antioxidative properties of human milk: A prospective controlled clinical investigation. J. Matern. Fetal Neonatal Med. 2015, 28, 734-738. [CrossRef] [PubMed]

66. Grune, T.; Lietz, G.; Palou, A.; Ross, A.C.; Stahl, W.; Tang, G.; Thurnham, D.; Yin, S.A.; Biesalski, H.K. Beta-carotene is an important vitamin A source for humans. J. Nutr. 2010, 140, S2268-S2285. [CrossRef] [PubMed]

67. Jiang, J.; Xiao, H.; Wu, K.; Yu, Z.; Ren, Y.; Zhao, Y.; Li, K.; Li, J.; Li, D. Retinol and $\alpha$-tocopherol in human milk and their relationship with dietary intake during lactation. Food Funct. 2016, 7, 1985-1991. [CrossRef] [PubMed]

(C) 2017 by the authors. Licensee MDPI, Basel, Switzerland. This article is an open access article distributed under the terms and conditions of the Creative Commons Attribution (CC BY) license (http://creativecommons.org/licenses/by/4.0/). 